\title{
Numerical Validation of Arecibo-Based Analytical Hat Antenna Theory Using Canonical PEC/PMC Strip Grids
}

\author{
Per-Simon Kildal \\ Department of Signals and Systems \\ Chalmers University of Technology \\ Gothenburg, Sweden \\ per-simon.kildal@chalmers.se
}

\author{
Abolfazl Haddadi \\ Department of Electrical Engineering \\ AmirKabir University of Technology \\ Tehran, Iran \\ abolfazl.haddadi@gmail.com
}

\author{
Jian Yang \\ Department of Signals and Systems \\ Chalmers University of Technology \\ Gothenburg, Sweden \\ jian.yang@chalmers.se
}

\begin{abstract}
The hat antenna is a self-supported rear-radiating waveguide feed used in the Comhat reflector that has been manufactured in more than $1 \mathrm{M}$ pieces for use in radio links. The design of hat feeds is done by numerical simulations taking advantage of its rotational symmetry. There exists an early analytical model for the far-field of the hat feed including the axial waveguide, and treating the hat brim as a PEC/PMC strip grid. The purpose of the present paper is to validate the theoretical model by simulations using COMSOL Multiphysics.
\end{abstract}

\section{INTRODUCTION}

The hat feed was invented during work with the upgrading of the radio telescope in Arecibo [1]. In order to get used to the spherical reflector geometry, Kildal presented a theoretical model for the old line feeds of the radio telescope [2], and by using this he could also see that it should be possible to illuminate a parabolic reflector with the same taper in $E-$ and $H$-planes so that the overall feed efficiency could be large with low cross-polar performance [3]. The feed efficiency is the first approximation to the aperture efficiency of the paraboloid, and it can be separated in many sub-efficiencies characterizing several different properties of the far-field, as explained in [4] and [5]. The hat-fed reflector (Figure 1a) was later been commercialized [6], [7] and has been manufactured in more than one million copies for microwave link antennas. It is known in the market as the Comhat antenna, after the start-up company Comhat $\mathrm{AB}$. The Comhat products are now the main products of LEAX Arkivator Telecom AB. It is also worth noting that the hat feed has a ring-shaped phase center [8].

The first 1987 paper on the hat feed presented an analytical formula for the far-field [3] based on [2]. This formula was the basic idea behind it. In practice the theoretical model has not been used since the 1980 s, because we instead tried to develop numerical models that even could provide the $\left|S_{11}\right|$. This was possible when the commercial QwickWave-V2D Solver became available in the late 1990s. This is still the most useful tool for its design. However, sometimes it is desirable to go back to the analytical roots of a design in order to see if it is possible to get a better fundamental understanding or its properties, and may be this can generate new ideas. Therefore,

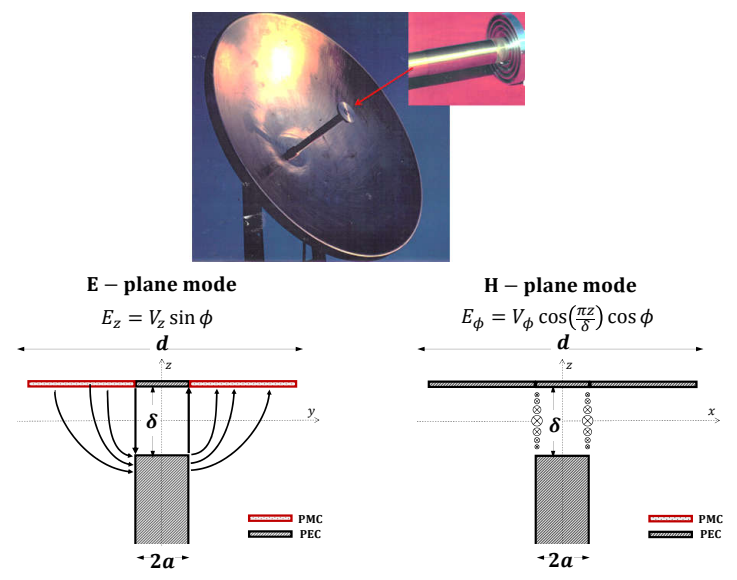

Fig. 1. a) Picture of hat-fed reflector antenna (early model of the Comhat antenna) and its corrugated hat feed. b) Drawing of the analysis model in COMSOL for $E$-plane mode, i.e. $z$-directed aperture field with PMC brim, and c) $H$-plane mode, i.e. $\phi$-directed aperture field and PEC brim. The early analytical mode was the same, but with an infinite diameter of the brim.

we have done this work with COMSOL that allows us to model rotationally symmetric structures, as well as PMCs of arbitrary shape, that were used to find the analytical far-field functions $(\mathrm{PMC}=$ Perfect Magnetic Conductor). The reason is that the corrugated brim of the hat feed was originally modeled as a soft surface, which we today describe as a transverse $\mathrm{PEC} / \mathrm{PMC}$ grid [9]. Introducing this in COMSOL, we also present a study of the effect of the diameter of the hat brim.

\section{Dual-Mode AnAlysis}

The early theoretical model was based on treating the field in the circumferential aperture in terms of two cylindrical parallel-plate modes, one with $z$-directed $H$-field, i.e. $\phi$-directed $E$-field, and another with $z$-directed $E$-field. These two modes were referred to as the $E$-plane mode and the $H$-plane mode [3]. The $E$-plane mode radiates both in $E$ - and $H$-planes, and the $H$-plane mode only in $H$-plane, when the cylinder is infinitely long. The PEC/PMC strip grid works as a PMC for the $E$-plane mode and as a PEC for the $H$-plane mode, as seen in figures $1 \mathrm{~b}$ and $1 \mathrm{c}$, respectively. 

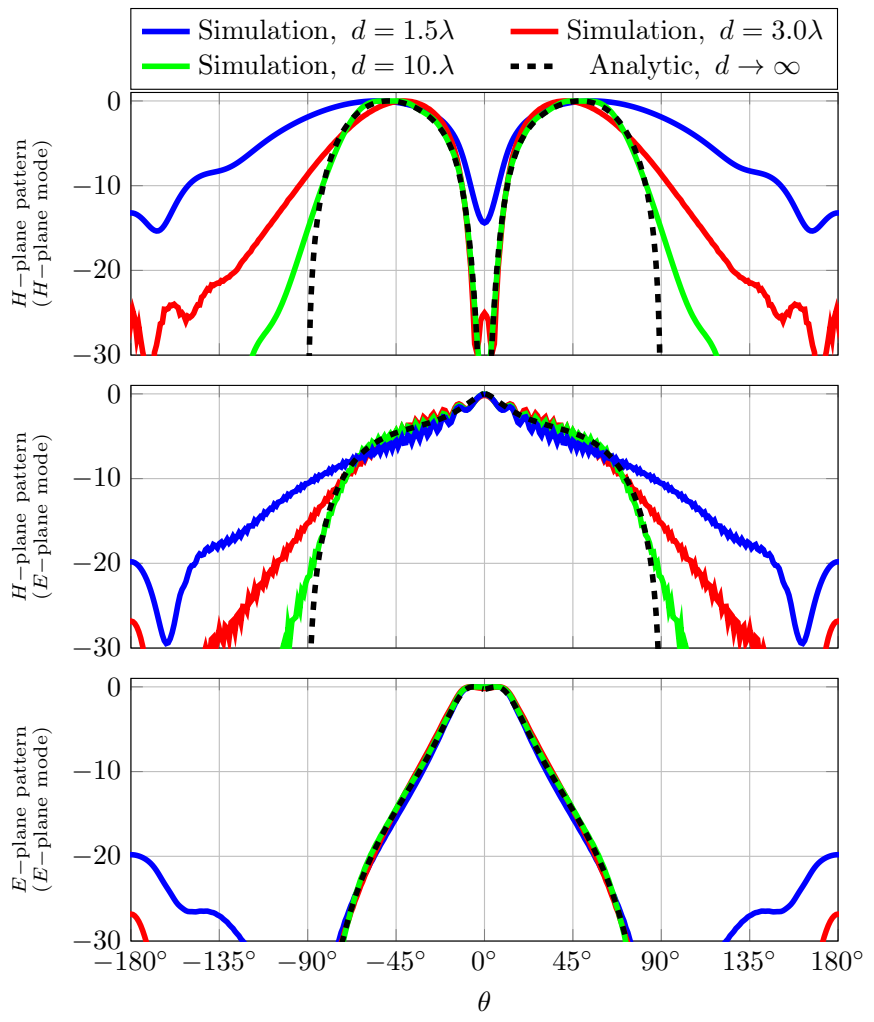

Fig. 2. Comparison between analytic (black dashed line) [3] and simulation (colored solid lines) results. (Top) $H$-plane pattern for $H$-plane mode, (middle) $E$-plane pattern for $E$-plane mode, and (bottom) $H$-plane pattern for $E$-plane mode. All simulations are done for $\delta=0.5 \lambda$, and $2 a=1 \lambda$.

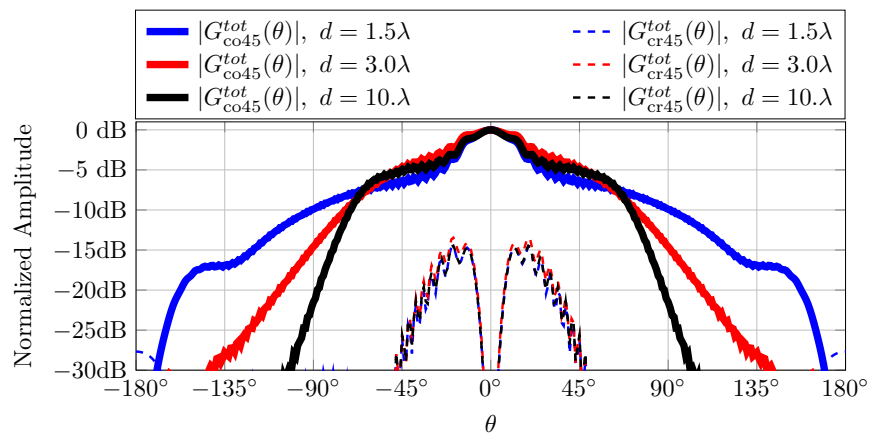

Fig. 3. Co- and cross-polar directive gain in $45^{\circ}$-plane for different hat brim diameters. The cross-polar pattern is forced to be zero at $\theta=55^{\circ}$ in all cases using a proper $V_{z} / V_{\phi}$. All simulations are done for $\delta=0.5 \lambda$, and $2 a=1 \lambda$. The length of the cylinder is $50 \lambda$.

The results for the $E-$ and $H$-plane modes using COMSOL are shown in Fig. 2. We see that they agree with the analytic results from [3] for large diameters $d$ of the brim.

The total far-field is obtained by adding the far-fields of the $E-$ and $H$-plane modes with the amplitude and phase excitation between them that provides the "best" illumination of the reflector. This was initially done in [3] by enforcing the cross-polar field to be zero at a specific $\theta$ angle, which is possible to enforce when all fields have BOR1 [5] azimuthal variations, i.e. the $\phi$-directed $E$-fields varies as $\cos \phi$, and the $\theta-$ and $z$-components of the $E$-field as $\sin \phi$. In Fig. 3 we have enforced the cross-polar field to be zero at $\theta=55^{\circ}$ for different hat brim diameters of $d=1.5 \lambda, 3 \lambda$, and $10 \lambda$. This appears when $\frac{V_{z}}{V_{\phi}} \approx 2 \angle 90^{\circ}$.

The hat feed is often used in deep $2 \times 90^{\circ}$ reflectors. We see that the co-polar fields in Fig. 3 have an illumination taper at $90^{\circ}$ of more than $20 \mathrm{~dB}$ if the brim has $10 \lambda$ diameter, $15 \mathrm{~dB}$ taper for $3 \lambda$ brim diameter, and $10 \mathrm{~dB}$ taper for $d=1.5 \lambda$. The aperture height used in these results is $\delta=0.5 \lambda$ and the outer diameter of the tube is $2 a=1 \lambda$. The highest cross-polar level in the $45^{\circ}$ - plane is about $-15 \mathrm{~dB}$. This will be reduced after the reflector.

\section{CONCLUSION}

We have presented a generic numerical model of the far-field of the hat feed based on the canonical PEC/PMC strip model. The results validate the original analytical model including the effect of the outer surface of the supporting waveguide. The COMSOL model has made it possible to make a generic study of the effect of the diameter of the brim.

This study has shown that basic generic studies using canonical surfaces, here in the form of a PEC/PMC strip grid, can be used to provide insight into the radiation mechanisms of practical antennas. Thus, concepts like canonical surfaces can be used together with general software like COMSOL for basic initial studies of new antenna types with artificial surfaces, in the same way as we in old days used analytical theories. The PEC and PMC play also an important role in the concept of gap waveguides [10]. There they are used as plates at a distance smaller than $\lambda / 4$ in order to create parallel-plate cut-off, and then this cut-off is used together with PEC strips to control the propagation in wanted directions between the plates.

\section{ACKNOWLEDGMENT}

The present work has been partly funded by the European Research Council via an advanced investigator grant ERC2012-ADG-20120216.

\section{REFERENCES}

[1] P.-S. Kildal, L. Baker, T. Hagfors et al., "Development of a dualreflector feed for the arecibo radio telescope: an overview," Antennas and Propagation Magazine, IEEE, vol. 33, no. 5, pp. 12-18, 1991.

[2] P.-S. Kildal, "Study of element patterns and excitations of the line feeds of the spherical reflector antenna in arecibo," IEEE Trans. on Anten. and Propag., vol. 34, no. 2, pp. 197-207, 1986.

[3] — "The hat feed: a dual-mode rear-radiating waveguide antenna having low cross polarization," IEEE Trans. on Anten. and Propag., vol. 35 , no. 9, pp. 1010-1016, 1987.

[4] _ _ "Factorization of the feed efficiency of paraboloids and cassegrain antennas," IEEE Trans. on Anten. and Propag., vol. 33, pp. 903-908, 1985.

[5] — Foundations of Antenna Engineering: A Unified Approach for Line-Of-Sight and Multipath. published by Kildal Antenn AB, 2015, (can be downloaded for free from http://www.kildal.se).

[6] J. Hansen, A. A. Kishk, P.-S. Kildal, and O. Dahlsjo, "High performance reflector hat antenna with very low sidelobes for radio-link applications," in Antennas and Propagation Society International Symposium, 1995. AP-S. Digest, vol. 2. IEEE, 1995, pp. 893-896.

[7] M. Denstedt, T. Östling, J. Yang, and P.-S. Kildal, "Tripling bandwidth of hat feed by genetic algorithm optimization," in Antennas and Propagation Society International Symposium. IEEE, 2007, pp. 2197-2200.

[8] J. Yang and P.-S. Kildal, "Calculation of ring-shaped phase centers of feeds for ring-focus paraboloids," IEEE Trans. on Anten. and Propag., vol. 48 , no. 4 , pp. 524-528, 2000. 
[9] P.-S. Kildal, A. Kishk, S. Maci et al., "Special issue on artificial magnetic conductors, soft/hard surfaces, and other complex surfaces," IEEE Trans. on Anten. and Propag., vol. 53, no. 1, pp. 2-7, 2005.

[10] A. Zaman and P.-S. Kildal, "Gap Waveguides," Chapter in Z. N Chen et al. (Eds.), Handbook of Antenna Technologies, Springer, 2016, [Online] Available: http://link.springer.com/referencework/10. 1007\%2F978-981-4560-75-7. 